\title{
Hypothermic circulatory arrest in operations on the thoracic aorta
}

\author{
Determinants of operative mortality and neurologic outcome
}

This study was undertaken to determine the factors that influence the final outcome after hypothermic circulatory arrest. Between 1985 and 1992 a uniform method of hypothermic circulatory arrest was used in 200 patients as the primary method of cerebral protection during operations on aneurysms of the thoracic aorta. There were 30 hospital deaths $(15 \%)$. Age greater than 60 years (relative risk 3.7, $p<0.02$ ), emergency operation and hemodynamic compromise (relative risk $22.2, p<0.000$ ), concomitant procedures (relative risk $2.7, p<0.04$ ), presentation with new neurologic symptoms (relative risk $5.2, p<0.04$ ), and postoperative permanent neurologic deficits (relative risk $9.4, p<0.000$ ) were found to be significant predictors of operative mortality. A total of 183 patients were available for evaluation of neurologic function and outcome. Multivariate analysis of this cohort of patients by multiple logistic regression showed that temporary neurologic dysfunction occurred in 36 cases (19\%). Temporary neurologic dysfunction correlated with the duration of hypothermic circulatory arrest $(47 \pm 16 \mathrm{~min}$ utes; odds ratio $1.06 /$ minute; $p<0.001)$ and age $(66 \pm 14$ years; odds ratio $1.07 /$ year; $p<0.001)$. Embolic strokes occurred in 22 patients $(11 \%)$ and were associated with permanent deficits in 13 $(7 \%)$. Strokes correlated significantly with age (older than $60,21 \%$ versus younger than $60,1 \%$; $p<0.001$ ) and operations on the arch and descending aortic aneurysms containing clot or atheroma $(p<0.001)$. This experience shows that the operative mortality is not affected by any parameters related to the use of hypothermic circulatory arrest. The incidence of temporary neurologic dysfunction rises linearly in relation to the age of the patient and the duration of hypothermic circulatory arrest. However, permanent neurologic injury is a result of thromboembolic events and is not related to the method of cerebral protection used. Additional methods to prevent perioperative embolic strokes are needed. Hypothermic circulatory arrest affords adequate cerebral protection if the arrest period is kept less than 60 minutes. We will continue to use this modality until the safety and utility of the alternate methods of cerebral protection are shown to be superior. (J THORAC CARDIOvaSC SuRG 1994;107:78899)

M. Arisan Ergin, MD, PhD, Jan D. Galla, MD, Steven L. Lansman, MD, PhD, Cid Quintana, MD, Carol Bodian, DrPH, ${ }^{a}$ and Randall B. Griepp, MD, New York, N.Y.

$\mathrm{T}_{\text {he }}$ he systematic application of profound hypothermia and circulatory arrest as a method of cerebral protection

From the Department of Cardiothoracic Surgery and the Department of Biomathematical Sciences, ${ }^{\text {a }}$ the Mount Sinai Medical Center, New York, N.Y.

Read at the Nineteenth Annual Meeting of The Western Thoracic Surgical Association, Carisbad, Calif., June 23-26, 1993.

Address for reprints: M. Arisan Ergin, MD, The Mount Sinai Medical Center, Department of Cardiothoracic Surgery, One Gustave L. Levy Place, Box 1028, New York, NY 10029.

Copyright $(1994$ by Mosby-Year Book, Inc.

$0022-5223 / 94 \$ 3.00+0 \quad 12 / 6 / 52311$ during replacement of the aortic arch was reported in 1975. ${ }^{1}$ Since then the indications for hypothermic circulatory arrest have been expanded to many procedures involving the thoracic aorta. Despite its prevalent clinical use the available information about the impact of this method on operative mortality and neurologic outcome is inadequate. The following study of our experience with the use of profound hypothermia and circulatory arrest in the treatment of thoracic aortic pathologic conditions was undertaken in an attempt to answer three fundamental questions: (1) does hypothermia, as applied, provide adequate protection for the central nervous system? (2) what 
are the safe limits of circulatory arrest? and (3) what other factors influence outcome? In the final analysis, particular emphasis was placed on the influence of the method per se on the operative mortality and the neurologic outcome.

\section{Patients and methods}

From September 1985 to September 1992, 200 consecutive patients underwent operations on the various portions of the thoracic aorta with the use of a standard technique of profound hypothermia and circulatory arrest as the only method of cerebral protection. There were 133 men and 67 women whose ages ranged from 26 to 85 years (mean $59.2 \pm 14.2$ years).

The surgical technique and the application of the profound hypothermic circulatory arrest throughout the study period were standard. The method of profound hypothermia and circulatory arrest has been previously described in detail. ${ }^{2,3}$ Brief$\mathrm{ly}$, it consists of core cooling during cardiopulmonary bypass to produce profound total body hypothermia to an average core temperature of $12^{\circ}$ to $15^{\circ} \mathrm{C}$ measured in the esophagus. The proximal repair is accomplished during this period of core cooling, which, in an average adult, requires at least 30 to 40 minutes of bypass cooling. The part of the procedure that necessitates the interruption of cerebral blood flow is then done during the period of circulatory arrest. During this time, the head is packed in ice to prevent warming of the central nervous system toward ambient temperatures. At the completion of the repair cardiopulmonary bypass is used to rewarm the patient and resuscitate the heart. Surface cooling, which was used as an integral part of the method earlier, was in more recent years reserved for only selected cases.

Myocardial protection was provided with single-dose cold potassium cardioplegia and continuous profound topical hypothermia in the pericardial well.

Standard doses of steroids were administered to all patients before circulatory arrest and for 48 hours after operation. Some patients in the early part of the study period also received barbiturates as an adjunct for cerebral protection.

All patients at the end of the procedure received blood components according to need. In the last 3 years of the study platelet-rich plasma was obtained in all patients in stable condition by plasmaphoresis. This plasma was reinfused at the end of the procedure, significantly aiding in hemostasis.

Surgical techniques were standard. End-to-end full-thickness open anastomoses with albumin-soaked and autoclaved grafts were used for most reconstructions. Inclusion techniques were reserved for cases in which difficulty with hemostasis was anticipated or in aortic root reconstructions when button coronary anastomoses were not possible. In some cases, inclusion techniques were supplemented with decompressive fistula into the right atrium. ${ }^{4}$ All patients received standard postoperative care.

A number of patient-related preoperative, operative, and postoperative factors were analyzed to determine their influence on the operative mortality and neurologic outcome (Appendix Table I).

Definitions. Emergency operations were defined as those procedures done within 16 hours of admission to the hospital or the establishment of the diagnosis. The remainder of the patients were operated on electively or urgently.

The presence of some perioperative conditions that were thought to be important contributors to either the operative mortality or the neurologic outcome were analyzed separately. These consisted of (1) preoperative hemodynamic compromise because of pericardial tamponade, shock, superior vena cava compression, or myocardial ischemia; (2) new preoperative neurologic symptoms and signs; (3) history of distant neurologic pathologic conditions such as carotid disease, stroke, or transient ischemic attack; (4) operative findings of rupture with either contained hematoma in the mediastinum or free rupture with blood in the pericardial or pleural spaces; and (5) the presence of loose clot or atheroma in the aorta significant enough to prompt the surgeon to make a special notation of it in the operative report.

For the purpose of analysis, the extent of replacement was simplified into three major groups according to the segment of aorta that dominated the repair. The group of proximal repair included all patients with ascending aortic and aortic root operations and the replacement of the proximal portion of the aortic arch. Arch replacement was defined as those procedures involving total replacement of the aortic arch with separate anastomoses for brachiocephalic vessels and the descending aorta with or without replacement of the varying portions of the ascending aorta, root, or the aortic valve. Procedures in these two groups were exclusively done through a median sternotomy, with right atrium and femoral artery cannulation. The distal arch and descending aorta group included all patients with replacement of varying portions of the descending aorta, the thoracoabdominal aorta, and the distal hemiarch, with or without total arch replacement. Procedures in this group were commonly done through a left thoracotomy, with femoral vein and femoral artery cannulation.

Patients who either died intraoperatively or did not survive long enough to determine their postoperative neurologic status were classified as early operative deaths. The remainder of the deaths were defined as hospital deaths. The overall operative mortality was defined as the sum total of these two groups.

To analyze neurologic outcome, we defined three distinct neurologic end points. First, temporary neurologic dysfunction was defined as the occurrence of postoperative confusion, agitation, delirium, prolonged obtundation, or transient parkinsonism without any localizing neurologic signs. Computed tomographic (CT) scans when done in these patients were usually normal. Second, permanent neurologic dysfunction was defined as the presence of permanent neurologic deficits that were focal (stroke) or global (parkinsonism, coma, gait disturbance) in nature and persisting at discharge from the hospital. When these permanent deficits were associated with strokes CT scans showed corresponding focal defects. Third, strokes were defined as transient or permanent neurologic deficits with localizing neurologic signs. These patients had corresponding new defects on CT scans.

Statistical methods. All variables were examined separately to determine their influence on the overall, early, and hospital mortality and on the three defined end points of neurologic outcome. Bivariate analysis was done for selected variables to examine their joint influence by the $\chi^{2}$ and Fisher's exact tests where appropriate. A $p$ value less than 0.05 was considered significant.

Variables that were determined to have significant influence on the mortality or the neurologic outcome by univariate or bivariate analysis were further subjected to multiple logistic regression analysis to determine the independent predictors of 
operative mortality and neurologic outcome. Odds ratios were calculated for each independent predictor as $\left(e^{b x}\right)$ where $b$ is the estimated regression coefficient corresponding to the variable $\mathrm{X}$.

\section{Results}

Pathologic and clinical classification of cases. A total of 101 patients had type A dissection ( 55 acute, 46 chronic), 10 had type B dissection ( 4 acute, 6 chronic), 44 had primarily atherosclerotic aneurysms, 31 had annuloaortic ectasia, 12 had iatrogenic dissections ( 4 acute, 8 chronic), and 2 had mycotic aneurysms. Twelve patients in this series also had Marfan's syndrome.

In 53 patients $(26.5 \%)$ the procedures were done on an emergency basis for ruptured or leaking aneurysms or acute dissections of the aorta. The remainder of the patients had elective or urgent operations.

The annual distribution of the cases and annual operative mortality in relation to the priority of the procedure are shown in Fig. 1.

A total of 129 patients had proximal repairs, including 79 open distal anastomoses and 50 proximal arch replacements; 75 of these patients had Bentall procedures. Total arch replacement with separate anastomosis for brachiocephalic vessels was done in 46 patients. In 29 patients the distal extent of the replacement was confined to the proximal portion of the descending aorta. These procedures were counted as arch replacements for purposes of analysis. The distal arch and descending aorta were the primary portions of the aorta replaced in 39 patients (Fig. 2). This group included 17 patients with arch replacements and 13 with distal arch replacements in combination with various portions of the descending aorta or the thoracoabdominal aorta. The total arch replacements in this latter group were done through bilateral thoracotomy in 11 and left thoracotomy in 3 patients. This extensive replacement was possible through a median sternotomy in only 4 of these patients.

Forty-eight concomitant procedures were done in addition to the primary aortic operation. The most common concomitant procedure was coronary artery bypass grafting in 19 patients, followed by mitral valve replacement in 5. The remaining 24 procedures included a host of miscellaneous procedures such as lobectomy, insertion of intraaortic balloon, subclavian artery bypass, and division of posterior aortic arch.

Overall mortality. Thirty patients died in the postoperative period for an overall mortality rate of $15 \%$ (30/ 200 ). Thirteen patients died in the early postoperative period ( 8 in the operating room; 5 shortly after leaving the operating room). The remaining 17 deaths occurred during the rest of the hospital stay (hospital deaths). The leading cause of death was cardiac in 17 (myocardial failure, postoperative myocardial infarction, cardiac rupture), bleeding in 5 , infection in 5 , distal aortic rupture in 1 , and redissection at the aortic root in 1 patient. Technical errors were contributing factors in 4 patients. Severe operation-related neurologic deficits were contributing factors in 3 deaths and 1 patient died of late massive intracerebral bleed.

Several factors proved to have significant influence on the overall mortality. Age greater than 60 years, emergency operation, presence of any of the predisposing preoperative or operative factors (rupture, preoperative neurologic symptoms, hemodynamic compromise, presence of clot or atheroma in the aorta), operation involving the descending aorta, incision other than a median sternotomy (especially bilateral thoracotomy), concomitant procedures, and postoperative occurrence of permanent dysfunction or stroke with permanent deficits were found to be statistically significant predictors of operative mortality. Pump time, myocardial ischemia time, cerebral ischemia time, and the level of hypothermia as reflected by the minimum core temperatures had no significant influence on the operative mortality. Occurrence of any major postoperative complication significantly increased the operative mortality with the exception of renal failure not requiring dialysis (Appendix Table I).

Four factors remained as independent predictors of overall mortality with significant relative risk factors calculated by logistic regression analysis: (1) emergency operation increased the risk by a factor of $2.8(p<0.02)$, (2) age greater than 60 years by a factor of $2.7(p<0.05)$, (3) presence of any predisposing preoperative condition by a factor of $3.5(p<0.01)$, and (4) concomitant procedures by a factor of $2.5(p<0.05)$. Because emergency operation and hemodynamic compromise are closely related, they proved to be the most dominant predictor of overall mortality when combined, increasing the relative risk by a factor of $22.2(p<0.0001)$.

Early mortality. Thirteen patients died before an adequate assessment of their neurologic function was possible.

Analysis of factors that influenced intraoperative or early postoperative death showed that age greater than 60 years was the most important discriminator $(1.1 \%$ in patients $<60$ years versus $10.9 \%$ in patients $>60$ years, $p<0.01)$. Accordingly, the influence of all other variables on early mortality was analyzed in the cohort of patients aged more than 60 years. Four factors were significantly associated with risk of early mortality. Early death occurred (1) in $37.5 \%$ of patients with rupture $(6 / 16, p<0.003)$, (2) in $46.2 \%$ of patients with hemodynamic compromise $(6 / 13, p<0.0001)$, (3) in $41.7 \%$ of patients with preoperative neurologic symptoms $(5 / 12$, 


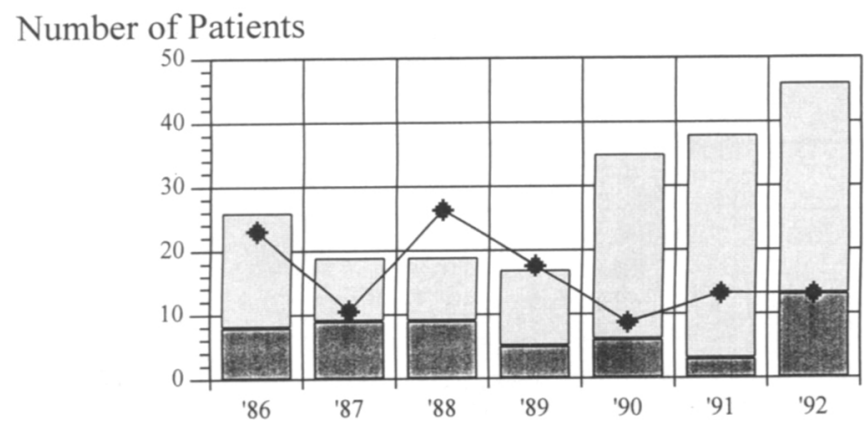

+ Percent Mortality
$\square$ Elective
$\square$ Emerg

Fig. 1. Annual distribution of cases (elective versus emergency [Emerg]) and percent yearly mortality; $n=200$, male/female ratio $133 / 67$, age $59.2 \pm 14.2$.

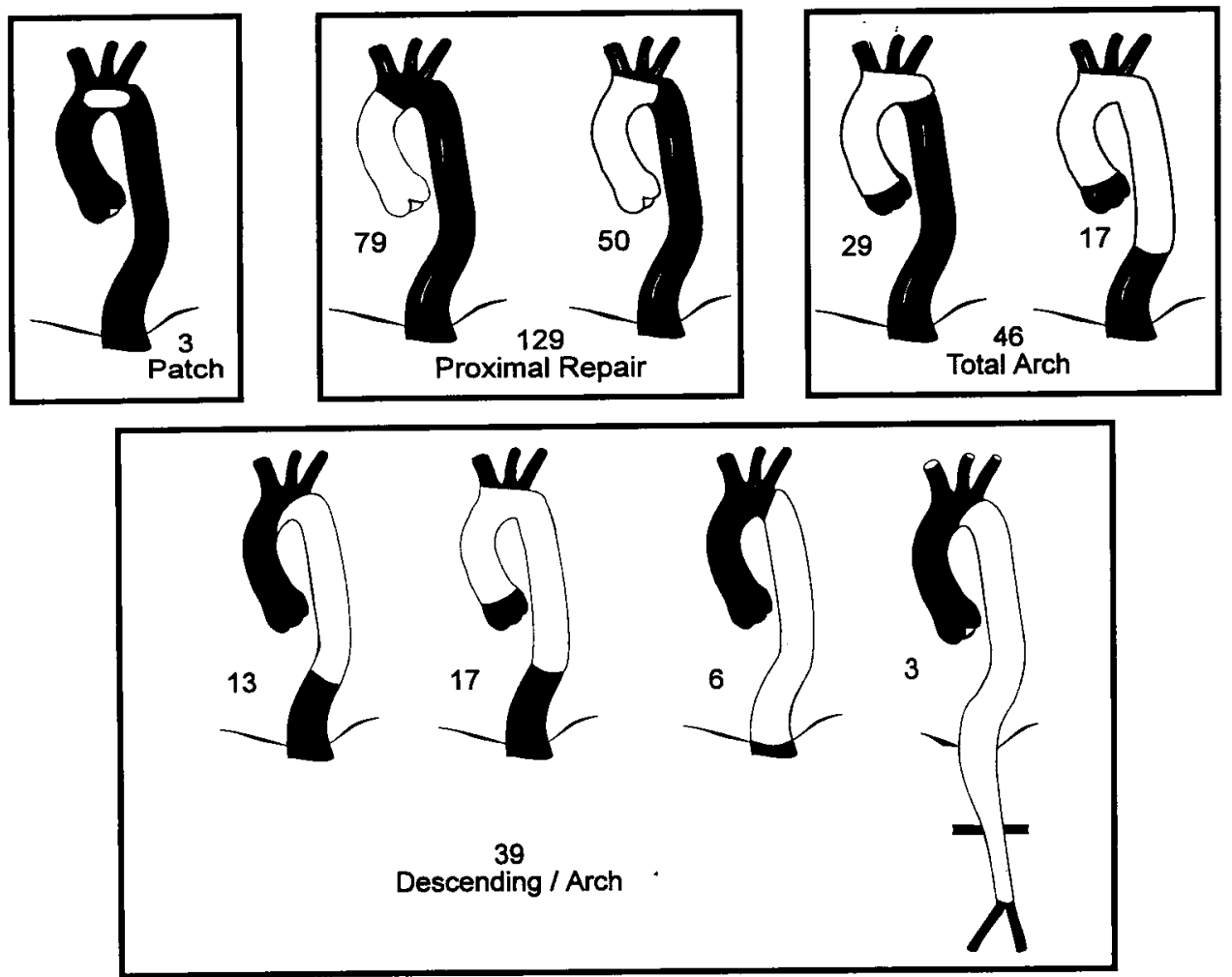

Fig. 2. Extent of replaced portion of aorta and related frequency.

$p<0.002$ ), and (4) in $24 \%$ of patients with descending aortic involvement $(6 / 25, p<0.03)$. All other factors including emergency operation, bypass time, myocardial ischemia time, cerebral ischemia time, and the level of hypothermia did not reach statistical significance.

The logistic regression analysis of these variables determined that besides age greater than 60 years only the presence of hemodynamic compromise and the presence of preoperative neurologic symptoms were independent predictors of early postoperative death. The relation of these factors to each other and the calculated relative risk factors for each are shown in Table I. Rupture and descending aorta distal arch replacement in different combinations approach statistical significance. However, they did not maintain significance once the presence of hemodynamic compromise was factored into the model.

Hospital mortality. A total of 187 patients survived the initial postoperative period and 170 patients were discharged from the hospital. Seventeen patients died in the hospital (hospital deaths). In this group of 187 survivors of the initial postoperative period, a thorough assessment of the neurologic function was possible. In this cohort of 


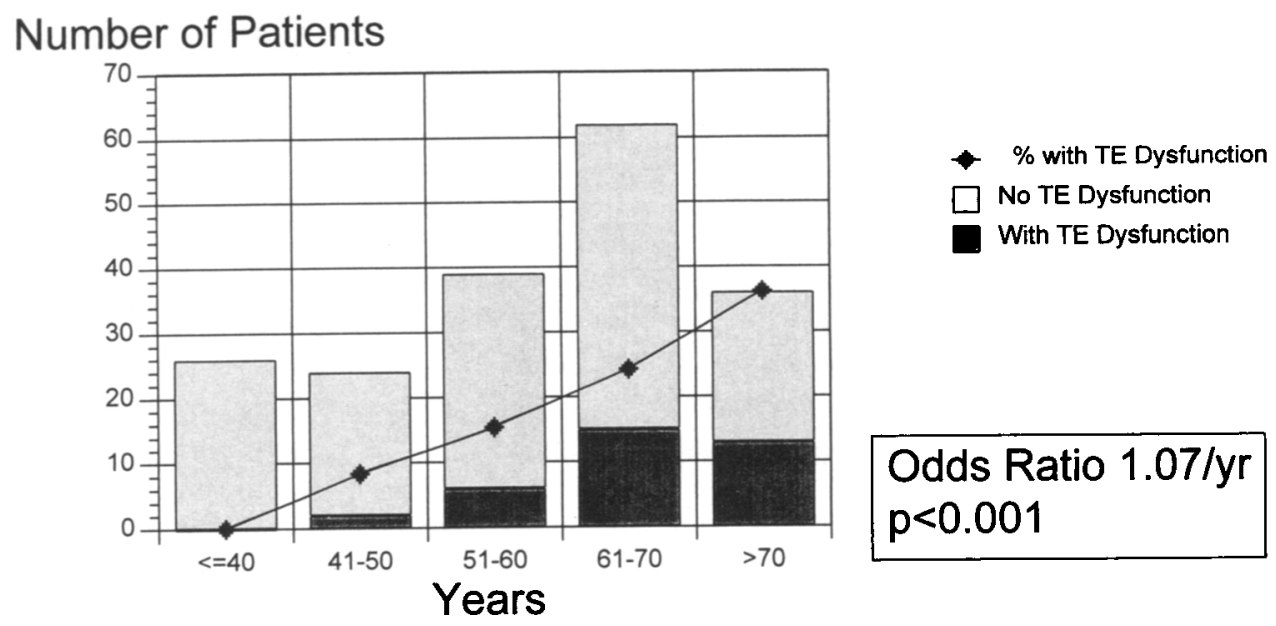

Fig. 3. Prevalence of temporary neurologic dysfunction as function of patient age. $T E$, Temporary.

Table I. Early death multivariate analysis (13/200 patients)

\begin{tabular}{lcc}
\hline \multicolumn{1}{c}{ Variable } & Relative risk $^{*}$ & p Value \\
\hline Hemodynamic compromise & 8.6 & 0.004 \\
Preop. neurologic symptoms & 5.2 & 0.039 \\
Rupture (free and contained) & 3.7 & 0.102 \\
Descending aorta resection & 3.3 & 0.113 \\
\hline
\end{tabular}

Logistic regression analysis. Note tendency of independent increase in risk for rupture and descending aorta resection.

*Relative risk calculated by logistic regression.

patients the occurrence of permanent neurologic injury emerges as an important predictor of hospital death. Six (46.1\%) of 13 patients with permanent neurologic deficit caused by stroke died in the hospital as compared with only $6.7 \%$ of patients without any neurologic injury.

Logistic regression analysis in this cohort of patients showed that the postoperative occurrence of permanent neurologic dysfunction (relative risk 9.4, $p<0.0003$ ) and the presence of hemodynamic compromise (relative risk $7.6, p<0.004)$ were the only independent predictors of hospital death. Addition of concomitant procedures approaches significance (relative risk $2.7, p<0.11$ ). There was, however, no additional predictive value obtained by considering age greater than 60 years, stroke, emergency operation, and extent of replacement. Again, other variables related to cardiopulmonary bypass, hypothermia, and circulatory arrest had no significant predictive value. A composite summary of all predictive factors for postoperative mortality is shown in Table II.

Neurologic outcome. Temporary neurologic dysfunction occurred in $19.3 \%(36 / 187)$ of patients who survived the initial postoperative period. On univariate and bivari- ate analysis age of the patient and the duration of the circulatory arrest emerged as the dominant factors predicting temporary neurologic dysfunction (Figs. 3 and 4). Other variables that were significantly associated with the occurrence of temporary neurologic dysfunction were arch dissection, type $B$ dissection, rupture, presence of any predisposing preoperative condition, hemodynamic compromise, clot or atheroma in the aorta, and descending aorta/distal arch operation. All other variables including bypass time and level of hypothermia had no predictive value.

By multiple logistic regression only three factors remained as independent predictors of temporary neurologic dysfunction: (1) age (odds ratio 1.07/year, $p<0.0001$ ), (2) circulatory arrest time (odds ratio 1.06/ minute, $p<0.0001$ ), and (3) rupture (relative risk 3.01, $p<0.001$ ). Other factors that were found to have significant influence on univariate or bivariate analysis lost their significance on multiple regression analysis.

Permanent neurologic injury was diagnosed in $9.6 \%$ $(18 / 187)$ of patients who survived the initial postoperative period. This injury was focal in nature and was due to embolic strokes in $6.9 \%(13 / 187)$. In $2.7 \%(5 / 187)$ the permanent injury was global and not embolic in nature as best as could be determined by cerebral CT scans.

On univariate and bivariate analysis emergency operation, type $\mathrm{A}$ dissection, rupture, preoperative neurologic symptoms, clot or atheroma in the aorta, and descending aorta/distal arch operation showed statistically significant association with the occurrence of permanent neurologic dysfunction.

By multiple logistic regression analysis preoperative neurologic symptoms (relative risk $4.0, p<0.026$ ), clot or atheroma in the aorta (relative risk 3.4, $p<0.02$ ), and 
Table II. Composite logistic regression analysis for mortality

\begin{tabular}{lcccccc}
\hline \multicolumn{1}{c}{ Variable } & $\begin{array}{c}\text { Early } \\
\text { risk* }\end{array}$ & $p$ Value & $\begin{array}{c}\text { Hospital } \\
\text { risk }\end{array}$ & p Value & $\begin{array}{c}\text { Overall } \\
\text { risk* }\end{array}$ & $p$ Value \\
\hline Age $>60$ yr & 10.9 & 0.01 & NS & NS & 3.7 & 0.02 \\
Emergency + hemodynamic compromise & 8.6 & 0.004 & 7.6 & 0.004 & 22.2 & 0.000 \\
Rupture & 3.7 & 0.102 & NS & NS & NS & NS \\
Concomitant procedure & NS & NS & 2.7 & 0.11 & 2.7 & 0.04 \\
Preop. neurologic symptoms & 5.2 & 0.04 & NS & NS & NS & NS \\
Descending aorta/arch & 3.3 & 0.11 & NS & NS & NS & NS \\
Permanent dysfunction & - & & 9.4 & 0.0003 & NS & NS \\
\hline
\end{tabular}

$N S$, Not significant.

*Relative risk calculated by logistic regression.

Table III. Multivariate analysis of permanent neurologic dysfunction

\begin{tabular}{lccccc}
\hline & \multicolumn{2}{c}{ Relative risk* } & & \multicolumn{2}{c}{$p$ Value } \\
\cline { 2 - 3 } \cline { 5 - 6 } Variable & Without desc. & With desc. & & Without desc. & With desc. \\
\hline Preop. neurologic symptoms & 4.0 & 5.3 & 0.05 & 0.026 \\
Clot or atheroma & 3.4 & 2.6 & 0.02 & 0.084 \\
Rupture (free and contained) & 3.0 & 2.5 & 0.04 & 0.099 \\
Descending aorta resection & & 3.1 & & 0.058 \\
\hline
\end{tabular}

Desc., Descending aorta/distal arch replacement.

*Odds ratio calculated by logistic regression.

rupture (relative risk $3.0, p<0.04$ ) were found to be independent predictors of permanent neurologic injury. Descending aorta/distal arch replacement seemed to be a significant contributing factor even though it did not attain statistical significance. Entering this factor into the model changed the relative risk ratios and the $p$ values of the last two independent predictors, which indicated that some of the predictive information that came from descending aorta/distal arch replacement was derived by its association with these two variables (Table III).

Embolic strokes were diagnosed in $11.8 \%(22 / 187)$ of patients who survived the initial postoperative period. This condition was transient in nature with recovery before discharge from the hospital in 4.8\% (9/187). In $6.9 \%(13 / 187)$ the strokes resulted in permanent neurologic deficits of varying intensity. All strokes were associated with corresponding defects seen on cerebral CT scans.

Age greater than 60 years was the single most important predictor of strokes ( $1.1 \%$ for age $<60$ years versus $21.4 \%$ for age $>60$ years; relative risk 19.1, $p<0.0001$; Fig. 5).

On univariate and bivariate analysis other significant associations between the occurrence of stroke and atherosclerotic aneurysms, presence of any predisposing preoperative condition, and clot or atheroma in the aorta were found. An interesting relation between extent of resection and occurrence of postoperative stroke was seen in the cohort of patients aged 60 years and older. Arch resection had an apparent preventive effect on the occurrence of stroke. This relation became clearer when analysis was controlled for the presence of clot or atheroma in the aorta. In the presence of clot or atheroma, the prevalence of postoperative stroke was equally high when only proximal repair or descending aorta replacement was done ( $44.4 \%$ versus $45.5 \%$ ), whereas it was significantly lower when the arch was replaced $(9.1 \%)$. This relation was shown to be statistically significant according to the Mantel-Haenszel test of association ( $p<0.04$; Table IV). - By multiple logistic regression analysis only two variables were found to be independent predictors of stroke: (1) age greater than 60 years (relative risk 19.1, $p<0.0001$ ) and (2) clot or atheroma in the aorta (relative risk 3.4, $p<0.04$ ). Replacement of the arch had a negative predictive value (relative risk $0.1, p<0.04$; Table V).

\section{Discussion}

Since its original description for replacement of the aortic arch the indications for use of hypothermic circulatory arrest have been expanded to include many procedures on the thoracic aorta. ${ }^{3,5-8}$ The routine use of open distal anastomosis in acute dissections of the aorta has led to the recognition of the importance of primary tears located in the aortic arch and to the adoption of new strategies in dealing with acute dissections of the aortic 


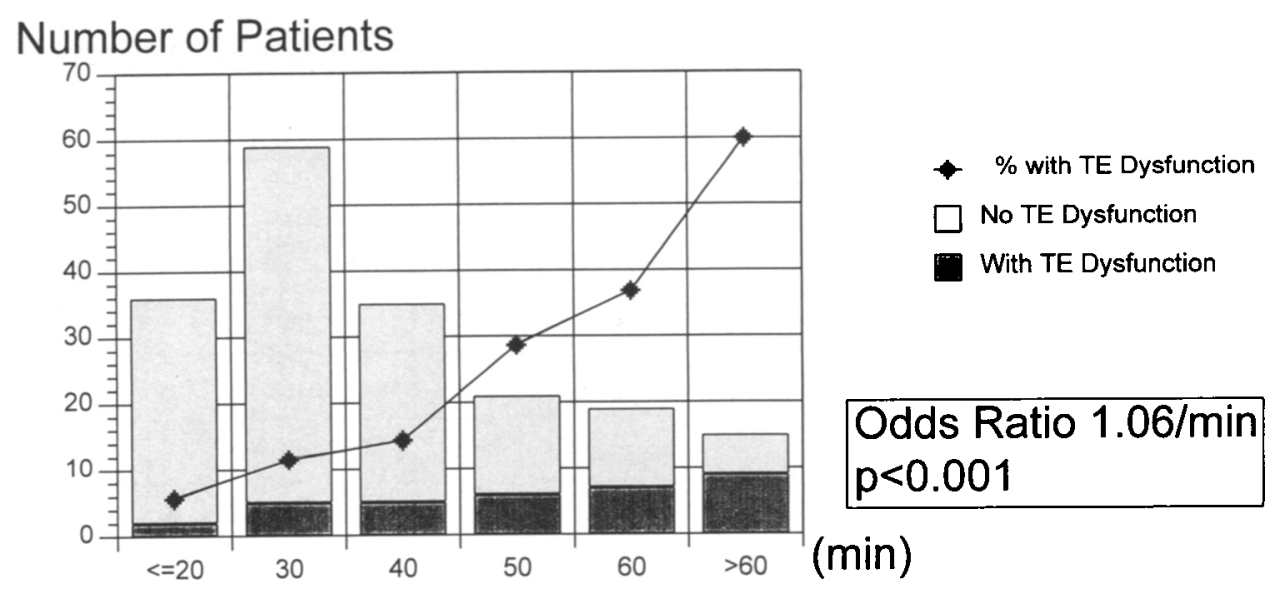

Fig. 4. Prevalence of temporary neurologic dysfunction as function of duration of circulatory arrest time. TE, Temporary.

\section{Number of Patients}

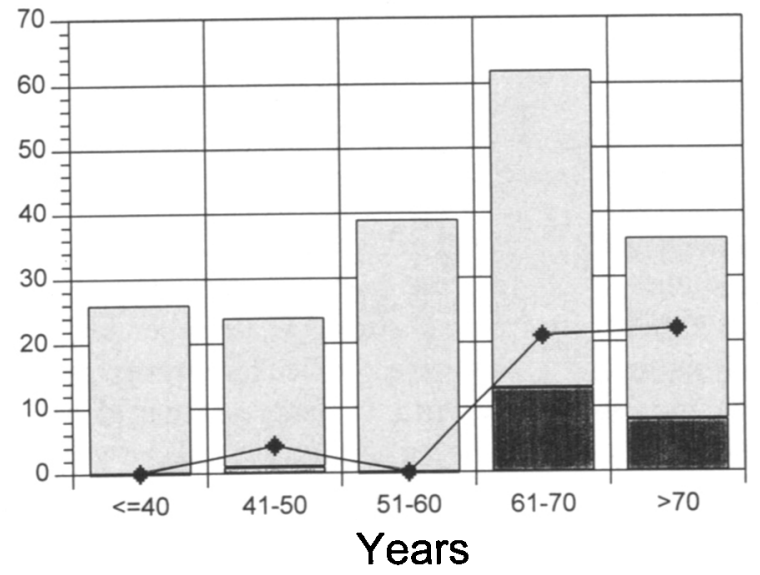

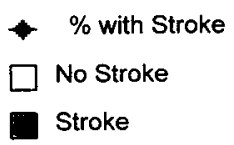

Odds Ratio 19.1 $\mathrm{p}<0.0001$

Fig. 5. Prevalence of stroke as function of patient age.

Table IV. Extent of replacement versus stroke (controlling for clot, atheroma $=$ yes)

\begin{tabular}{lrrrrrr}
\hline & \multicolumn{2}{c}{ No stroke } & & \multicolumn{2}{c}{ Stroke } \\
\cline { 2 - 3 } \cline { 6 - 7 } \multicolumn{1}{c}{ Variable } & No. & $\%$ & & No. & $\%$ \\
\hline Proximal repair & 5 & 55.6 & & 4 & 44.4 \\
Arch & 10 & 90.9 & & 1 & 9.1 \\
Descending aorta/arch & 6 & 54.6 & & 5 & 45.5 \\
\hline
\end{tabular}

Frequency table. $p<0.04$ by Mantel-Haenszel test of association.

arch. ${ }^{3,9-11}$ Hypothermic circulatory arrest has permitted operations on the distal arch or the descending aorta without application of proximal clamps. ${ }^{7}$ It has also been used as a primary means of preventing spinal cord injury during operations on the descending aorta. ${ }^{8}$

Among the most cited disadvantages of the technique
Table V. Multivariate analysis of stroke

\begin{tabular}{lcl}
\hline \multicolumn{1}{c}{ Variable } & Relative risk* & p Value \\
\hline Arch replacement & 0.1 & 0.04 \\
Clot or atheroma & 3.4 & 0.04 \\
Age $>60$ & 19.1 & 0.0001 \\
\hline
\end{tabular}

Logistic regression analysis.

*Odds ratio calculated by logistic regression.

are hypothermia-associated coagulopathy, prolongation of the procedure, and the time limitation imposed by the circulatory arrest..$^{12,13}$ This has led to the exploration of alternative methods of cerebral protection in recent years. ${ }^{11,14,15}$ Clear definition of the actual impact of the method of cerebral protection on the operative mortality and the final neurologic outcome is essential for continued clinical utility of any technique. The body of experience 
with hypothermic circulatory arrest is large enough to allow the search for the answers to the fundamental questions regarding the effectiveness and safety of the technique.

The overall mortality rate of $15 \%$ observed in this group of patients compares favorably with that in other similar series. Laas and associates ${ }^{10}$ reported an overall mortality rate of $13.9 \%$ for arch replacement with profound hypothermia and circulatory arrest. Their reported mortality rate varied from a low of $6.9 \%$ for chronic dissections to a high of $20.5 \%$ for acute dissections. Svensson and colleagues ${ }^{16}$ recently reported an operative mortality rate of $10 \%$ in 656 patients treated with hypothermic circulatory arrest. The $10.8 \%$ mortality rate observed in elective procedures of the present series is comparable with these. These figures for hypothermic circulatory arrest methods can be compared with the operative mortality seen with conventional perfusion techniques. Moreno-Cabral and associates ${ }^{17}$ reported a mortality rate of $9.7 \%$ in 175 patients from Stanford, and Crawford and associates ${ }^{18}$ reported $9 \%$ in 717 patients. The Stanford series did not include arch resections. All patients were treated with conventional perfusion methods. In the series of Crawford and associates ${ }^{18} 44 \%$ of the patients were treated with methods other than hypothermic circulatory arrest. Galloway and colleagues ${ }^{19}$ reported a mortality rate ranging from $17.9 \%$ to $12.3 \%$ in 165 patients with ascending aortic and aortic arch aneurysms. Again, in that series, $41 \%$ of patients were treated with methods other than circulatory arrest.

We observed a sharp increase in mortality in patients older than 60 years of age. The influence of age was also reported by others as the only constant determinant of mortality. ${ }^{16-19}$ Besides age, the other predictors of mortality in our experience were emergency operation and presence of preoperative hemodynamic instability. The $26.4 \%$ operative mortality rate for emergency procedures in the present series compares favorably with the $32 \%$ to $60 \%$ mortality rates for emergency operations reported by others. ${ }^{17,} 19$ We found that the presence of hemodynamic compromise was a stronger independent predictor of overall mortality than the urgency of the procedure. In the presence of preoperative hemodynamic compromise the operative mortality increased by a factor of 7.6 to 22.2 for all phases of the hospital stay (Table II). On the other hand, contained or free rupture found at operation did not significantly influence operative mortality once the presence of hemodynamic compromise was factored into the model by multivariate analysis. These findings indicate that the substantial risk associated with the emergency procedure is mainly determined by the presence of hemodynamic instability.
Among the neurologic factors, two were found to have strong influences on postoperative mortality. Presence of new preoperative neurologic symptoms predicted early postoperative death and the occurrence of postoperative permanent neurologic deficit was a strong predictor of hospital death. Similar observations were made by Svensson and colleagues. ${ }^{16}$ Laas and associates ${ }^{10}$ reported neurologic complications as the second most important cause of hospital mortality in their series.

The extent of the aortic replacement in the present series had no significant influence on operative mortality. No clear-cut association between extent of the operation and mortality was seen in the series of Crawford ${ }^{18}$ or Svensson ${ }^{16}$ and their associates, whereas arch replacement was reported to be associated with significantly increased operative risk by Galloway and colleagues. ${ }^{19}$

Contrary to early reports on operation for thoracic aneurysms, ${ }^{20}$ we were unable to find a significant effect on mortality of any of the perfusion parameters including bypass time, myocardial ischemia time, cerebral ischemia time, and the level of hypothermia.

The prevalence of the major complications was quite similar to those of the reported larger series. ${ }^{7-19}$ The occurrence of any major postoperative complication, cardiac complications leading the list, was associated with a significant increase in mortality. This is consistent with the earlier reported experiences. ${ }^{10,18}$

These considerations led us to conclude that the operative mortality primarily is determined by the pathologic condition and patient characteristics rather than the method of cerebral protection. Improvement in the operative mortality is dependent on modifications of these preoperative factors. A concerted effort, especially toward the reduction of emergency procedures by aggressive and timely elective treatment of aneurysms, may have a substantial impact on the operative mortality.

Neurologic complications have been the cornerstone of the debate concerning operations on the thoracic aorta in general and the arch of the aorta in particular. ${ }^{11-14,16} \mathrm{~A}$ careful look at the neurologic outcome in this series showed several important findings. The symptom complex that we defined as temporary neurologic dysfunction is quite similar to the one occasionally observed in elderly patients after other types of routine cardiac operations. ${ }^{21,22}$ This was thought to be related to "alterations in cerebral micro circulation or gaseous micro emboli" by some. ${ }^{12}$ This form of neurologic dysfunction was the most common neurologic problem that we encountered (18\%). It is rarely mentioned by others reporting on thoracic aortic surgery. ${ }^{12}$ In our experience, the occurrence of temporary dysfunction unequivocally is related to advanced age and the duration of the circulatory arrest 
(calculated odds ratios 1.07/year and 1.06/minute, respectively; Figs. 3 and 4). The cardiopulmonary bypass time and level of hypothermia had no predictive value. Comparable clinical information in the present literature is not available. The temporary neurologic dysfunction may be a manifestation of transient neurologic injury. Its linear relation to the arrest time points toward a safe limit beyond which the protective effect of hypothermia as applied in these patients, especially in older age groups, is open to question. Despite its frequent occurrence temporary neurologic dysfunction had no lasting effects and had no influence on mortality.

In contrast, the occurrence of permanent neurologic dysfunction was a major determinant of operative mortality in this series and in those reported by others. ${ }^{10,12,16,18}$ Permanent neurologic deficits were seen in 18 patients $(9 \%)$. In 13 of these patients the permanent deficits were related to embolic strokes. In three patients the neurologic deficit was clearly a major contributor to the hospital mortality.

Clearly embolic strokes occurred in $11.8 \%(22 / 187)$ of the patients; they were transient in $4.8 \%$ and associated with permanent deficits in $6.9 \%$ of the patients. Almost an identical prevalence of strokes (7\%) with permanent deficits was reported by Svensson and colleagues ${ }^{16}$ in 656 patients. In our experience age greater than 60 years and the presence of clot or atheroma in the aorta were the most important predictors of postoperative stroke. This is supporting evidence that indicates the role of particulate embolization in the pathogenesis of these strokes. An important relation between the extent of the replacement of the aorta and occurrence of stroke was also observed. In the presence of extensive atheromatous disease or significant clot in the aorta, total arch replacement reduced the prevalence of stroke significantly compared with proximal repair or descending aorta and distal arch replacements. This finding can be interpreted as showing that the removal of all diseased aorta from the proximal to the cerebral circulation may have a protective value against embolic strokes. This in turn may have important implications for devising strategies for prevention of perioperative strokes in patients with heavily calcified or atheromatous proximal aortas who are undergoing cardiac operations for indications other than aneurysmal disease. Whether it is justified to project this finding to other situations is open to speculation.

The embolic origin of the strokes dictates the adoption of several preventive principles during the conduct of the procedure. In patients with extensive atheromatous disease of the descending aorta and the arch, retrograde perfusion of the aorta should be avoided as much as pos- sible. Especially in descending aortic and distal arch operations the diseased aorta should not be manipulated during the cooling period. A period of retrograde cerebral perfusion at the end of the arrest period may be a useful adjunct to wash out debris that may have escaped distally into the cerebral circulation. ${ }^{23} \mathrm{At}$ the end of the arrest period and after the retrograde washout of the cerebral circulation, bypass should always be resumed antegradely through the newly inserted prosthetic graft. This is important to prevent embolization from distal sources in the remaining thoracoabdominal aorta and debris that may have escaped into these areas at the earlier phases of the procedure. The effectiveness of these measures in preventing strokes remains to be seen with increasing clinical experience.

\section{Conclusions}

In light of the results of this study, we believe that the following conclusions are justified. Operative mortality is not related to the use of hypothermic circulatory arrest. It is a function of the pathologic condition and other predetermined patient-related factors. Temporary neurologic dysfunction is related to the cerebral ischemia time. This relation is markedly pronounced in older patients. The substantial increase in its incidence beyond $60 \mathrm{~min}$ utes of circulatory arrest may indicate an upper limit beyond which the adequacy of cerebral protection with the current methods is questionable. Arrest times exceeding 60 minutes are not advisable, especially in elderly persons. Hypothermic circulatory arrest is an effective method of cerebral protection within the constraints of this time period. We will continue to use this technique until the safety and utility of alternate methods of protection have been proved. Permanent neurologic dysfunction is not related to the use of hypothermic circulatory arrest. It is, in general, the result of embolic strokes. Further refinements of current techniques and new methods are needed for prevention of embolic strokes.

\section{REFER E N CES}

1. Griepp RB, Stinson EB, Hollingsworth JF, Buehler D. Prosthetic replacement of the aortic arch. J THORAC CARDIOVASC SURG 1975;70:1051-63.

2. Ergin MA, Griepp RB. Progress in treatment of the aneurysms of the aortic arch. World J Surg 1980;4:535-43.

3. Ergin MA, O'Connor J, Guinto R, Griepp RB. Experience with profound hypothermia and circulatory arrest in the treatment of aneurysms of the aortic arch: aortic arch replacement for acute arch dissections. J THORAC CARDIOVASC SURG 1982;84:649-55.

4. Cabrol C, Pavie A, Mesnildrey P, et al. Long-term results 
with total replacement of the ascending aorta and reimplantation of the coronary arteries. J THORAC CARDIOVASC SURG 1986;91:17-25.

5. Livesay JJ, Cooley DA, Duncan JM, Ott DA, Walker WE, Reul GJ. Open aortic anastomosis: improved results in the treatment of aneurysms of the aortic arch. Circulation 1982;66(Suppl):I1 22-7.

6. Crawford ES, Crawford JS, Safi HJ, Coselli JS. Redo operations for recurrent aneurysmal disease of the ascending aorta and transverse aortic arch. Ann Thorac Surg 1985;40:439-55.

7. Crawford ES, Coselli JS, Safi HJ. Partial cardiopulmonary bypass, hypothermic circulatory arrest, and posterolateral exposure for thoracic aortic aneurysm operation. J THORAC CARDIOVASC SURG 1987;94:824-7.

8. Kouchoukos NT, Wareing TH, Izumoto H, Klausing W, Abboud N. Elective hypothermic cardiopulmonary bypass and circulatory arrest for spinal cord protection during operations on the thoracoabdominal aorta. J THORAC Cardiovasc Surg 1990;99:659-64.

9. Lansman SL, Raissi S, Ergin MA, Griepp RB. Urgent operation for acute transverse aortic arch dissection. $\mathrm{J}$ Thorac Cardiovasc SuRG 1989;97:334-41.

10. Laas J, Jurmann MJ, Heinemann M, Borst HG. Advances in aortic arch surgery. Ann Thorac Surg 1992;53:227-32.

11. Bachet J, Teodori G, Goudot B, et al. Replacement of the transverse aortic arch during emergency operations for type A acute aortic dissection: report of 26 cases. J THORAC CARDIOVASC SURG 1988;96:878-86.

12. Livesay JJ, Cooley DA, Reul GJ, et al. Resection of aortic arch aneurysms: a comparison of hypothermic techniques in 60 patients. Ann Thorac Surg 1983;36:19-28.

13. Cooley DA, Ott DA, Frazier OH, Walker WE. Surgical treatment of aneurysms of the transverse aortic arch: experience with 25 patients using hypothermic techniques. Ann Thorac Surg 1981;32:260-72.

14. Frist WH, Baldwin JC, Starnes VA, et al. A reconsideration of cerebral perfusion in aortic arch replacement. Ann Thorac Surg 1986;42:273-81.

15. Ueda Y, Miki S, Kusuhara K, Okita Y, Tahata T, Yamanaka K. Surgical treatment of aneurysm or dissection involving the ascending aorta and aortic arch, utilizing circulatory arrest and retrograde cerebral perfusion. J Cardiovasc Surg 1990;31:553-8.

16. Svensson LG, Crawford ES, Hess KR, Coselli JS, Safi HJ. Deep hypothermia and circulatory arrest: determinants of stroke and early mortality in 656 adult patients. J THORAC Cardiovasc Surg 1993;106:19-31.

17. Moreno-Cabral CE, Miller DC, Mitchell RS, et al. Degenerative and atherosclerotic aneurysms of the thoracic aorta: determinants of early and late surgical outcome. J THORAC CARDiovasc Surg 1984;88:1020-32.

18. Crawford ES, Svensson LG, Coselli JS, Safi HJ, Hess KR. Surgical treatment of aneurysm and/or dissection of the ascending aorta, transverse aortic arch, and ascending aorta and transverse aortic arch: factors influencing survival in
717 patients. J THORAC CARDIOvaSC SURG 1989;98:65974.

19. Galloway AC, Colvin SB, LaMendola CL, et al. Ten-year operative experience with 165 aneurysms of the ascending aorta and aortic arch. Circulation 1989;80(Suppl):I249-56.

20. Liddicoat JE, Bekassy SM, Rubio PA, Noon GP, DeBakey ME. Ascending aortic aneurysms: review of 100 consecutive cases. Circulation 1975;52(Suppl):I202-9.

21. Tufo HM, Ostfeld AM, Shekelle R. Central nervous system dysfunction following open heart surgery. JAMA 1970;212:1333-40.

22. Shaw PJ, Bates D, Cartlidge NEF, et al. Early neurological complications of coronary artery bypass surgery. BMJ 1985;291:1384-7.

23. Yamashita C, Nakamura H, Nishikawa $Y$, Yamamoto S, Okada M, Nakamura K. Retrograde cerebral perfusion with circulatory arrest in aortic arch aneurysms. Ann Thorac Surg 1992;54:566-8.

\section{Discussion}

Dr. Julie Swain (Las Vegas, Nev.). This study represents the most important contribution to the literature on the effect of heart surgery on the brain that I have seen. This study will remain the gold standard for neurologic sequelae after circulatory arrest for some time to come.

The completely classified clinical status of these patients and the critical and careful analysis of the data are unprecedented. In particular, the definition of operative mortality as in-hospital mortality, rather than as the 30-day mortality used in some other large series, is important. Likewise, the careful classification of temporary and permanent defects, especially the inclusion of postoperative confusion and agitation, is important in that these findings have been neglected by those who report large series with no neurologic defects. This again emphasizes that quality of data is more important than quantity of data.

I have two questions. First, the authors showed a $20 \%$ temporary neurologic dysfunction rate that was proportional to circulatory arrest time and concluded that times longer than 60 minutes should be avoided. Because this group has excellent technical expertise such that it is clear that times longer than 60 minutes are occasionally needed, what plans do you have to change your cerebral protection strategies to protect the patients who need more than 60 minutes of circulatory arrest?

Dr. Ergin. I would like to thank Dr. Swain for her kind remarks. Her question is an important one. Time limitation imposed by the arrest period has been the Achilles heel of the method. However, if the operation is well planned, one rarely will have to exceed 60 minutes of arrest time. In cases in which longer arrest times are anticipated, the connection to the brachiocephalic vessels is done first and antegrade hypothermic low-flow perfusion of this system is resumed as described by Frist and associates from Stanford (Ann Thorac Surg 1986;42:273-81.). In our experience this was rarely ever necessary.

Dr. Swain. Second, two thirds of the patients with permanent neurologic deficits had evidence of embolic strokes, as did $12 \%$ of the total patients. Do you think that retrograde perfusion would decrease this number of embolic strokes and are you currently using this modality? 
Dr. Ergin. New methods are needed for prevention of embolic strokes. Retrograde cerebral perfusion may be useful for washing out embolic debris from the cerebral circulation. We believe the other important point in the prevention of strokes is avoiding retrograde perfusion of the aorta as much as possible, especially in patients with disease of the descending aorta. As soon as the new graft is inserted, reperfusion should be begun through the graft in an antegrade manner, rather than resuming perfusion through the femoral artery. We have adopted these two methods as measures for prevention of embolic strokes.

Dr. John E. Connolly (Irvine, Calif.). We are certainly dealing with a surgical group of pioneers in the area, and I do not pretend to have the clinical experience they do. However, we have had a lot of laboratory experience that I think throws some light on their use of circulatory arrest, and I would like to reiterate some of the things that I think are applicable.

More than 30 years ago, we, along with Dr. Kimoto in Tokyo, worked in the laboratory and clinically on selective cerebral hypothermia and later on total circulatory arrest with deep hypothermia and concluded that the latter is more easily done and is safer than selective cerebral hypothermia.

I am sure that Dr. Griepp and his colleagues are familiar with the dog experiments that led to our ideas on this. We took 80 dogs and had them trained by psychologists so that we later could not only assess neurologic damage under the microscope but also be sure that the animals could still perform certain higher functions. We found that when brain temperatures of $20^{\circ} \mathrm{C}$ were reached we could perform 1 hour and 45 minutes of circulatory arrest without clinical neurologic changes; however, we did see subtle microscopic changes in the brains. With 2 hours of circulatory arrest at $20^{\circ} \mathrm{C}$ brain temperature, we observed severe neurologic changes. At 1 hour of circulatory arrest with brain temperature at $20^{\circ} \mathrm{C}$, we observed no microscopic changes and the animals were still able to do the tricks that they had been conditioned to perform. We concluded that brain damage with profound hypothermia and circulatory arrest is not caused by the hypothermia per se, as the authors agree, but rather by inadequate protection from cerebral anoxia during a given period of arrest.

I think the most important lesson that we learned was to know what the exact brain temperature was at the time of arrest. Brain temperature lags well behind esophageal temperature and this explains why short periods of cooling, which were associated with early neurologic complications such as those reported by Björk years ago, cast a pall over this technique originally. I believe very strongly that one must know the brain temperature to make this technique absolutely safe and to know exactly how long one can work under circulatory arrest. We have recommended monitoring the brain temperature with a fine thermistor probe introduced through a very tiny twist drill that will just admit the needle into the cerebral hemisphere. We see no complications with the needle either in the dog or in the human being. If you do not want to put a needle thermistor probe into the brain, some researchers have monitored neural temperature by monitoring the cerebrospinal fluid temperature. I have not tried this. A brain temperature of $20^{\circ} \mathrm{C}$ in the dog is safe for an hour. I think most of us who have worked on dogs realize that the dog is less tolerant of most things than the human being, and so I think we can extrapolate that an hour is safe at that temperature. In cases that might require more than an hour of arrest, although our experiments did not look at temperatures below $20^{\circ} \mathrm{C}$, perhaps if the brain temperature were cooled below $20^{\circ} \mathrm{C}$, one could expect to be able to work for longer periods of arrest.

Finally, the embolic complications mentioned with aortic arch operations are similar to those we see in abdominal aortic operations. Those of you doing peripheral vascular operations know to clamp the iliac arteries before manipulating the aorta or the aneurysm and to carefully clean out the atheroma of the open vessel by flushing. Likewise, those who do carotid endarterectomy know the internal carotid artery should be clamped before one manipulates or clamps any of the other carotid vessels. I trust that the authors take into account all of those maneuvers to minimize their risk of embolism.

The final thing that I would like to ask the authors is whether they are sure that the so-called embolic neurologic deficits are truly embolic and not possibly caused by prolonged ischemia without a properly protected brain and cord?

Dr. Ergin. Thank you, Dr. Connolly. It is a privilege for us to have you discuss this paper. Actually I think you were one of the discussants of Dr. Griepp's original paper in 1975. We are well aware of your contributions to the field.

The embolic nature of these permanent injuries that we see and label as strokes are proved by CT scans. If results of a CT scan were normal in a patient with a permanent neurologic deficit, we labeled that as a global dysfunction and assumed that it was due to global injury to the brain caused by ischemia rather than local embolic phenomena.

Dr. Denton A. Cooley (Houston, Tex.). I would like to ask Dr. Ergin about ischemic spinal cord injury. Do you consider it necessary to reimplant segmental vessels in thoracoabdominal aneurysms, or do you believe that the deep hypothermia provides sufficient protection of the spinal cord during periods of circulatory interruption?

Dr. Ergin. As you have shown in the past, hypothermia is the only proven method that provides adequate across-the-board protection of the spinal cord. However, the final result still is dependent on the identification and reattachment of the critical intercostal arteries for continued perfusion of the cord. In this series, hypothermic circulatory arrest was not used as a method of cord protection. None of these patients had intercostal reimplantations. One patient, with a descending aortic aneurysm, had postoperative paraplegia.

Dr. Cooley. There have been reports of series of cases in which the entire descending and abdominal aorta was resected under deep hypothermia without neurologic complications and without reimplantation of the segmental vessels.

Dr. Ergin. I believe Dr. Kouchoukos' report is the largest of its kind. But, as I said, paraplegia developed in one of our patients.

Dr. Thomas Wareing (St. Louis, Mo.). I, too, would applaud Dr. Ergin and Dr. Griepp and their associates for continuing to be advocates and leaders in this modality of hypothermic circulatory arrest. It is certainly a remarkable experience they are reporting and we all realize that this modality has allowed us to extend treatment to a number of people with difficult thoracic aortic problems and other cardiac surgical problems.

I noticed the authors did not mention much about the technique of pharmacologic manipulation during the period of circulatory arrest and I wondered if Dr. Ergin would comment on that. Also we have discussed the possibility of ischemic injury and embolic injury as being the genesis of many of these neurologic problems, but there is also some experimental evidence accumulating currently that is implicating the toxic effects of 
Appendix Table Ia. Univariate analysis for overall mortality

\begin{tabular}{|c|c|c|c|c|}
\hline Variable & $\begin{array}{c}\text { No. of } \\
\text { patients }\end{array}$ & $\begin{array}{l}\% \text { of } \\
\text { total }\end{array}$ & $\begin{array}{c}\% \\
\text { dead }\end{array}$ & $p$ Value \\
\hline All patients & 200 & 100 & 15 & - \\
\hline \multicolumn{5}{|l|}{ General } \\
\hline Age $>60$ & 110 & 55 & 20.9 & 0.04 \\
\hline Age $<60$ & 90 & 45 & 7.8 & - \\
\hline Male & 133 & 66.5 & 12.8 & 0.22 \\
\hline Female & 67 & 43.5 & 19.4 & - \\
\hline Emergency & 53 & 26.5 & 26.4 & 0.02 \\
\hline Reoperation & 41 & 20.5 & 14.6 & 0.94 \\
\hline \multicolumn{5}{|l|}{ Pathologic condition } \\
\hline Type A dissection & 55 & 27.5 & 16.4 & 0.74 \\
\hline Arch dissection & 17 & 8.5 & 23.5 & 0.30 \\
\hline Type B dissection & 4 & 2 & 25.0 & 0.57 \\
\hline Atherosclerotic & 44 & 22 & 18.2 & 0.50 \\
\hline Rupture (free) & 25 & 12.5 & 44.0 & 0.000 \\
\hline \multicolumn{5}{|l|}{ Preoperative factors } \\
\hline Hemodynamic compromise & 20 & 10.0 & 60.0 & 0.000 \\
\hline Preop. neurologic symptoms & 17 & 8.5 & 52.9 & 0.000 \\
\hline Clot/atheroma & 48 & 24 & 25.0 & 0.026 \\
\hline \multicolumn{5}{|l|}{ Surgical factors } \\
\hline Proximal repair & 129 & 64.5 & 10.1 & - \\
\hline Arch & 29 & 14.5 & 10.3 & - \\
\hline Descending aorta/arch & 39 & 19.5 & 33.3 & 0.001 \\
\hline Concomitant procedure & 48 & 24.0 & 25.0 & 0.013 \\
\hline Incision non-median sternotomy & 33 & 16.5 & 33.3 & 0.001 \\
\hline Bilateral thoracotomy & 11 & 5.5 & 54.6 & 0.000 \\
\hline \multicolumn{5}{|l|}{ Neurologic function } \\
\hline Temporary dysfunction & 36 & 18.0 & 5.6 & 0.412 \\
\hline Permanent dysfunction & 18 & 9.0 & 38.9 & 0.000 \\
\hline Stroke & 22 & 11.0 & 27.3 & 0.002 \\
\hline Stroke with permanent dysfunction & 13 & 6.5 & 46.15 & 0.000 \\
\hline \multicolumn{5}{|l|}{ Complications } \\
\hline Postop. bleeding & 19 & 9.5 & 47.8 & 0.000 \\
\hline Respiratory insufficiency & 54 & 27.0 & 29.6 & 0.000 \\
\hline Tracheostomy & 32 & 16 & 31.3 & 0.000 \\
\hline Renal failure with dialysis & 9 & 4.5 & 66.7 & 0.000 \\
\hline Renal failure without dialysis & 10 & 5.0 & 30.0 & 0.173 \\
\hline Cardiac complications & 68 & 34.0 & 23.5 & 0.000 \\
\hline Infection & 37 & 18.5 & 35.1 & 0.000 \\
\hline Any complication & 115 & 57.5 & 19.3 & 0.000 \\
\hline
\end{tabular}

excitatory neurotransmitters such as glutamate and some others as perhaps propagating this sort of injury. What is the authors' opinion about that information?

Dr. Ergin. There is a large body of pharmacologic, neurologic, and surgical literature addressing both questions that you have raised. Time limitation does not allow me to respond in detail. We have not changed the main methods of pharmacologic management that were reported before. We have stopped using barbiturates completely. We continue to use steroids. We do not use any other agents for scavenging free radicals and counteracting effects of other toxic metabolic by-products of ischemia and reperfusion. When such agents are transported from experimental to clinical use, they certainly will be adjunct to the method.

\section{Appendix Table Ib}

\begin{tabular}{ccccc}
\hline $\begin{array}{c}\text { Perfusion } \\
\text { parameters }\end{array}$ & Mean & STD & Min. & Max. \\
\hline $\begin{array}{l}\text { Bypass time (min) } \\
\text { Myocardial ischemia }\end{array}$ & 185.8 & 68.9 & 77 & 511 \\
$\quad \begin{array}{l}\text { (min) } \\
\begin{array}{c}\text { Cerebral ischemia } \\
\text { (min) }\end{array}\end{array}$ & 35.8 & 16.5 & 12 & 80 \\
$\begin{array}{c}\text { Esophageal temp. } \\
\left({ }^{\circ} \mathrm{C}\right)\end{array}$ & 14.2 & 2.9 & 7.9 & 22.7 \\
Rectal temp. $\left({ }^{\circ} \mathrm{C}\right)$ & 19.9 & 3.4 & 10 & 31.5 \\
\hline
\end{tabular}

$S T D$, standard deviation. 